\title{
mTOR and PDGF Pathway Blockade Inhibits Liver Metastasis of Colorectal Cancer by Modulating the Tumor Microenvironment
}

\author{
Ryo Yuge, ${ }^{*}$ Yasuhiko Kitadai, ${ }^{*}$ Kei Shinagawa, ${ }^{\dagger}$ Mieko Onoyama, ${ }^{*}$ Shinji Tanaka, ${ }^{\dagger}$ Wataru Yasui, ${ }^{\ddagger}$ and Kazuaki Chayama*
}

\author{
From the Departments of Gastroenterology and Metabolism* and Molecular Pathology, ${ }^{\ddagger}$ Graduate School of Biomedical and Health Sciences, Hiroshima \\ University, Hiroshima; and the Department of Endoscopy, ${ }^{\dagger}$ Hiroshima University Hospital, Hiroshima, Japan
}

\author{
Accepted for publication \\ October 14, 2014. \\ Address correspondence to \\ Yasuhiko Kitadai, M.D., Ph.D., \\ Department of Gastroenter- \\ ology and Metabolism, Grad- \\ uate School of Biomedical and \\ Health Sciences, Hiroshima \\ University, 1-2-3 Kasumi, \\ Minami-ku, Hiroshima \\ 734-8551, Japan. E-mail: \\ kitadai@hiroshima-u.ac.jp.
}

\begin{abstract}
Tumor growth and metastasis are not determined by cancer cells alone but also by a variety of stromal cells, and platelet-derived growth factor receptors (PDGF-Rs) are overexpressed by various stromal cell populations. Activation of PI3K-AKT-mTOR signaling is frequently observed in many cancer types. We investigated whether the mTOR inhibitor everolimus, alone or in combination with the PDGF-R tyrosine kinase inhibitor nilotinib, can inhibit growth and metastasis of human colon cancer. The effects of nilotinib and everolimus on tumor growth and metastasis were examined in an orthotopic mouse model of human colon cancer and a model of liver metastasis. After treatment with nilotinib (versus distilled water), the stromal reaction of xenografts growing in the cecal wall and liver was significantly decreased. After treatment with everolimus, the stromal reaction did not decrease, but tumor cell proliferation and microvessel density decreased. With the two drugs in combination, both stromal reaction and tumor cell proliferation decreased and apoptosis of tumor cells increased, resulting in remarkable inhibition of tumor growth at both the orthotopic and the metastatic site. Concurrent inhibition of tumor cells and activated stromal cells by a PDGF-R tyrosine kinase inhibitor and an mTOR inhibitor used in combination may represent a novel therapeutic approach for colorectal cancer. (Am J Pathol 2015, 185: 399-408; http://dx.doi.org/10.1016/j.ajpath.2014.10.014)
\end{abstract}

Colorectal cancer (CRC) is the third most commonly diagnosed cancer worldwide and the third leading cause of cancer death in Japan. ${ }^{1}$ Despite a growing number of chemotherapeutic agents, the median survival of patients with metastatic CRC remains less than 20 months, which underscores the urgent need for novel therapeutic approaches. ${ }^{2}$ Recent molecular and cellular biology studies have shown that tumor growth and metastasis are not determined by cancer cells alone, but also by a variety of stromal cells. The stroma constitutes a large part of most solid tumors, and cancer-stromal cell interaction contributes functionally to tumor growth and metastasis. ${ }^{3,4}$ Tumor stroma contains many different cell types, including activated fibroblasts (myofibroblasts), endothelial cells, and inflammatory cells. Activated fibroblasts in cancer stroma are prominent modifiers of tumor progression and thus are called carcinoma-associated fibroblasts (CAFs). ${ }^{5}$

We have previously shown in human colon cancer tissues that platelet-derived growth factor receptors (PDGF-Rs) are overexpressed by various stromal cell populations, including CAFs and pericytes, but not by the cancer cells themselves. ${ }^{6,7}$ PDGFs, on the other hand, are expressed by colon cancer cells. ${ }^{5,7}$ PDGF-R signaling is reported to be involved in pericyte recruitment ${ }^{8,9}$ and in controlling interstitial fluid pressure in stroma, influencing transvascular transport of chemotherapeutic agents in a paracrine manner. ${ }^{10}$ Blockade of PDGF-R signaling pathways in tumor-associated stromal cells with the use of a PDGF-R tyrosine kinase inhibitor (imatinib or nilotinib) has been shown to inhibit the stromal reaction in an orthotopic mouse model of human colon cancer and a gastric cancer xenograft model. $^{11}$

\footnotetext{
Supported in part by grants-in-aid for cancer research from the Ministry of Education, Culture, Science, Sports and Technology of Japan.

Disclosures: Everolimus and nilotinib were provided by Novartis Pharma K.K. (Tokyo, Japan).
} 
Activation of phosphoinositide 3-kinase-protein kinase B-mammalian target of rapamycin signaling (PI3K-AKTmTOR) is associated with growth and progression of a number of cancers, including CRC. ${ }^{12,13}$ mTOR is a central regulatory kinase that increases the production of proteins that stimulate key cellular processes such as cell proliferation, metabolism, survival, and angiogenesis. ${ }^{14}$ Everolimus, an oral inhibitor of the serine-threonine kinase mTOR, which functions downstream of the PI3K-AKT pathway, has been shown in cultured tumor cells to have antiangiogenic effects via reductions in expression of hypoxia-inducible factor-1 (HIF-1) and vascular endothelial growth factor (VEGF). ${ }^{15-19}$ We therefore investigated whether nilotinib and/or everolimus inhibits the growth and metastasis of CRC in an orthotopic mouse model of CRC and in an experimental liver metastasis model.

\section{Materials and Methods}

\section{Surgical Specimens}

Archival formalin-fixed, paraffin-embedded tumor tissues were obtained from Hiroshima University Hospital. The specimens, from 27 patients who underwent surgical resection for colon cancer, were examined by immunohistochemistry. Patient privacy was protected in accordance with the Ethical Guidelines for Human Genome/Gene Research of the Japanese Government. Informed consent was not required, and all personally identifiable information was removed before analysis of the tissue samples.

\section{Human Colon Carcinoma Cell Lines and Culture Conditions}

Human colon cancer cell lines KM12SM ${ }^{20}$ and KM12C were a kind gift of Dr. Isaiah J. Fidler (University of Texas, Houston, TX). The KM12SM cell line is a highly metastatic clonal cell line selected from the parental KM12C cell line. DLD1 and WiDr human colon cancer cell lines and the human osteosarcoma cell line MG63 (which served as a positive control for PDGF-R expression) were obtained from the Health Science Research Resources Bank (Osaka, Japan). These cell lines were maintained in Dulbecco's modified Eagle's medium with $10 \%$ fetal bovine serum (Sigma-Aldrich, St. Louis, MO) and a penicillin-streptomycin mixture. The cultures were maintained for no longer than 12 weeks after recovery of cells from frozen stock.

\section{Reagents}

Nilotinib (Tasigna) and everolimus (Afinitor) were kindly provided by Novartis Pharma (Tokyo, Japan). Nilotinib was dissolved in $0.5 \%$ hydroxypropylmethylcellulose aqueous solution containing $0.05 \%$ Tween 80 at a concentration of 4 $\mathrm{mg} / \mathrm{mL}$, then diluted in sterile water. Everolimus was diluted in sterile water for oral administration. Primary antibodies were as follows: polyclonal rabbit anti-PDGF-R $\beta$, polyclonal rabbit anti-phosphorylated PDGF-R $\beta$, polyclonal rabbit anti-human VEGF-A, and polyclonal rabbit anti-PDGFBB subunit (Santa Cruz Biotechnology, Dallas, TX); rat anti-mouse CD31 (BD Pharmingen; BD Biosciences, San Diego, CA); monoclonal mouse anti-desmin antibody from (Invitrogen; Life Technologies, Carlsbad, CA); rabbit anti- $\alpha$-smooth muscle actin ( $\alpha$-SMA) (Abcam, Cambridge, UK); Ki-67 equivalent antibody (Novocastra; Leica Microsystems, Newcastle upon Tyne, UK); polyclonal rabbit anti-mouse type I collagen (Novotec, Saint Martin La Garenne, France); and monoclonal rabbit anti-mouse S6 ribosomal protein antibody and monoclonal rabbit antiphosphorylated mouse S6 ribosomal protein antibody (Cell Signaling Technology, Danvers, MA). Fluorescent secondary antibodies were Alexa Fluor 488-conjugated goat anti-rabbit IgG, Alexa Fluor 488-conjugated goat anti-rat IgG, and Alexa Fluor 546-conjugated goat anti-rabbit IgG (Invitrogen; Life Technologies).

\section{Western Blot Analysis}

Cultured colon cancer cells and MG63 cells were washed and then scraped into phosphate-buffered saline containing 5 $\mathrm{mmol} / \mathrm{L}$ EDTA and $1 \mathrm{mmol} / \mathrm{L}$ sodium orthovanadate. Pellets obtained by centrifugation were resuspended in a lysis buffer (20 mmol/L Tris-HCl, pH 8.0; $137 \mathrm{mmol} / \mathrm{L} \mathrm{NaCl} ; 10 \%$ glycerol; $2 \mathrm{mmol} / \mathrm{L}$ EDTA; $1 \mathrm{mmol} / \mathrm{L}$ phenylmethylsulfonyl fluoride; $20 \mathrm{mmol} / \mathrm{L}$ leupeptin; $0.15 \mathrm{unit} / \mathrm{mL}$ aprotinin) and centrifuged; the supernatant was then collected. Tumors growing in nude mice were resected and homogenized in a lysis buffer. The protein contents of the samples were quantified spectrophotometrically, as described previously. ${ }^{21}$

\section{Growth Inhibition Assays}

Equal numbers of KM12SM cells were seeded onto 12-well plates at a density of $1 \times 10^{5}$ cells per well in the appropriate culture medium with supplements. Cells were treated with various doses of everolimus and nilotinib (including an equivalent clinically effective $\operatorname{dose}^{22}$ ), and growth curves were generated with the use of a brightfield image label-free, high-content time-lapse assay system (IncuCyte; Essen BioScience, Ann Arbor, MI) that automatically measured cell confluence as a percentage over a 5-day period.

\section{Animals and Orthotopic Implantation of Tumor Cells}

Female athymic BALB/c nude mice were obtained from Charles River Japan (Tokyo, Japan). The mice were maintained under specific pathogen-free conditions and used at 8 weeks of age. The study was conducted with permission from the Committee on Animal Experimentation of Hiroshima University. To produce cecal tumors, PDGF-expressing $\mathrm{KM} 12 \mathrm{SM}$ cells $\left(5 \times 10^{5}\right.$ cells $)$ in $50 \mu \mathrm{L}$ of Hanks' balanced salt solution were injected into the cecal wall of the 


\section{A}

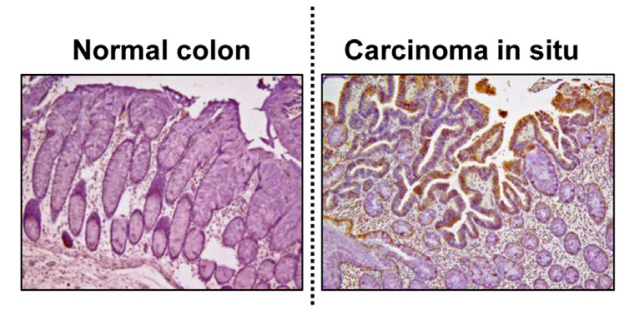

B

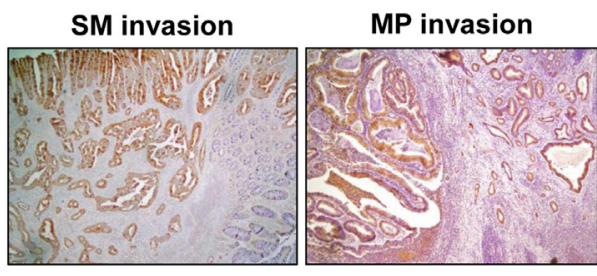

C

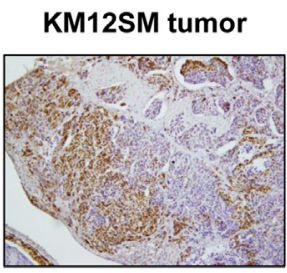

KM12C tumor

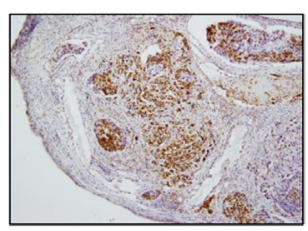

KM12SM DLD1 WiDr KM12C

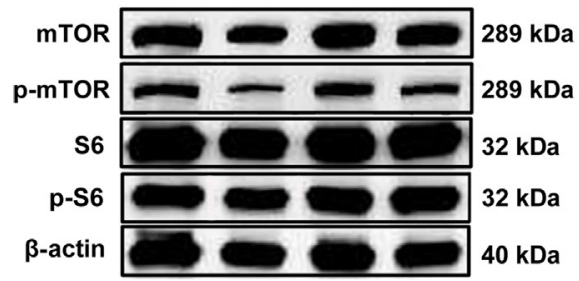

D

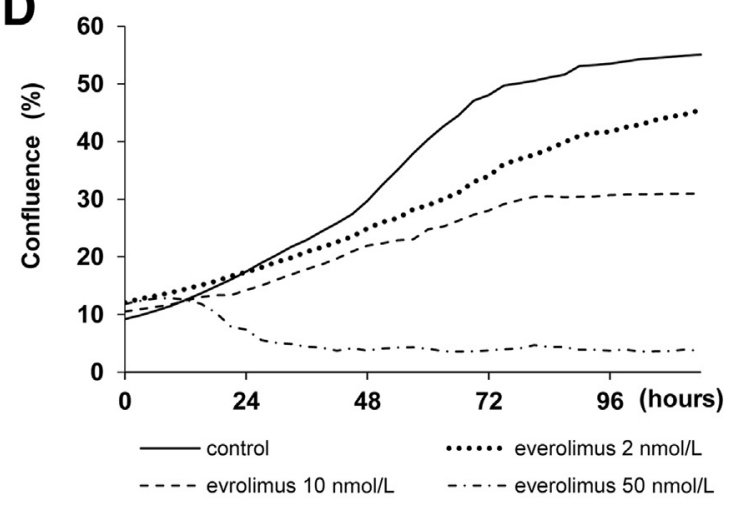

E

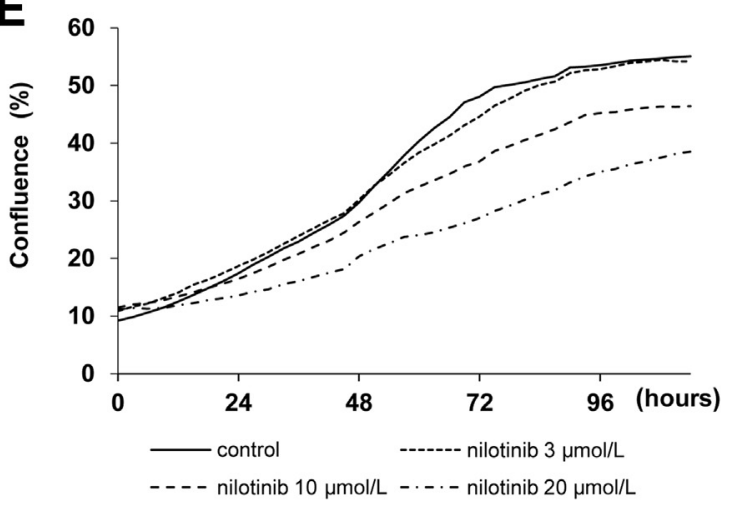

Figure 1 A-C: Expression of p-S6 in normal colon tissue versus human colorectal cancer (CRC) tissue (A), orthotopic KM12 tumors (B), and CRC cell lines (C). A: Normal colon mucosa showed no immunoreactivity for $\mathrm{p}-\mathrm{S6}$, but diffuse cytoplasmic staining was seen in tumor cells. B: Phosphorylation of S6 protein was detected in orthotopic xenografts derived from KM12SM and KM12C human colon cancer cells. C: Expression and phosphorylation of mTOR and S6 proteins was detected in all four CRC cell lines. D and E: The growth of KM12SM cells with and without everolimus (D) or nilotinib (E) treatment was measured using IncuCyte technology, which monitors cell confluency in real time (one image per 3 hours) for a period of 5 days. MP, muscularis propriae; SM, submucosal.

nude mice under zoom stereomicroscope observation. To produce experimental liver metastasis, $1 \times 10^{6} \mathrm{KM} 12 \mathrm{SM}$ cells in $50 \mu \mathrm{L}$ of Hanks' balanced salt solution were injected into the spleen of nude mice. ${ }^{6}$

\section{Treatment of Established Human Colon Cancer Tumors Growing in Nude Mice Cecum or Liver}

At 14 days after cell implantation, mice were randomly assigned to four treatment groups: i) daily administration of water by oral gavage (control), ii) daily oral gavage of 100 $\mathrm{mg} / \mathrm{kg}$ nilotinib, iii) daily oral gavage of $1 \mathrm{mg} / \mathrm{kg}$ everolimus, and iv) daily oral gavage of $100 \mathrm{mg} / \mathrm{kg}$ nilotinib and $1 \mathrm{mg} / \mathrm{kg}$ everolimus. Animal numbers were $n=10$ per group in the orthotopic model and $n=5$ per group in the liver metastatic model. Treatments lasted 28 days.

\section{Necropsy and Histological Studies}

Mice bearing orthotopic tumors were euthanized with diethyl ether. Body weights were recorded. After necropsy, tumors were excised and weighed. For immunohistochemistry, one part of the tumor tissue was fixed in formalin-free immunohistochemistry zinc fixative (BD Pharmingen; BD Biosciences, San Diego, CA) and embedded in paraffin; the other part was embedded in Tissue-Tek OCT compound (Sakura Finetek, Torrance, CA), rapidly frozen in liquid nitrogen, and stored at $-80^{\circ} \mathrm{C}$. All macroscopically enlarged regional (celiac and para-aortal) lymph nodes were harvested, and the presence of metastatic disease was confirmed by histological examination. Tumor volume was calculated as $V=1 / 2\left(\right.$ length $\times$ width $\left.^{2}\right)$.

\section{Immunohistochemical Staining and Immunofluorescence Double Staining}

Formalin-fixed, paraffin-embedded tissues cut into serial $4-\mu \mathrm{m}$ sections were used for immunohistochemistry. The procedures for immunohistochemical detection of $\alpha$-SMA, type I collagen, Ki-67, phosphorylated S6 ribosomal protein (p-S6), or VEGF-A were as described previously. ${ }^{23}$ Apoptotic cells in tissue sections were detected by TUNEL 


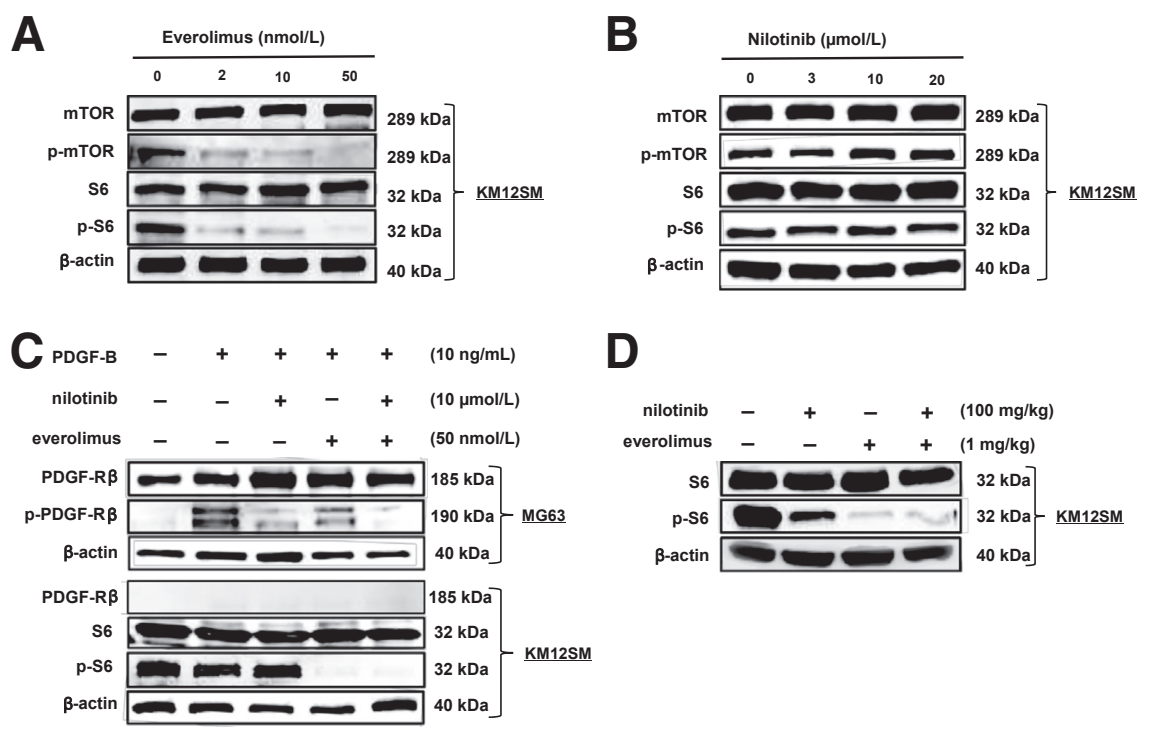

Figure 2 Effect of everolimus and/or nilotinib on $\mathrm{mTOR}$ and platelet-derived growth factor (PDGF) signaling. A: Treatment of KM12SM cells with everolimus for 48 hours resulted in a dosedependent decrease in $\mathrm{p}$-mTOR and p-S6 levels. B: Nilotinib had no significant effect on KM12SM cells. C: Nilotinib treatment resulted in a decrease of $p$-PDGF-R $\beta$ in MG63 cells. Expression of PDGF$R \beta$ was undetectable in KM12SM cells. Everolimus did not affect the PDGF pathways in MG63 cells. D: Phosphorylation of S6 in orthotopic KM12SM tumors in nude mice was inhibited by oral administration of everolimus and by nilotinib and everolimus in combination. assay with an ApopTag Plus peroxidase in situ apoptosis detection kit (Millipore-Chemicon International, Temecula, CA) according to the manufacturer's instructions. The transplanted tumor tissues were prepared into $10-\mu \mathrm{m}$ frozen sections and then were subjected to immunofluorescence analyses using the following primary antibodies: PDGF-R $\beta$, p-PDGF-R $\beta$, CD31, and desmin (for pericytes). The fluorescent signal of secondary antibody was captured by confocal laser-scanning microscopy (Carl Zeiss Microscopy, Thornwood, NY; Jena, Germany). Endothelial cells were identified by green fluorescence, and pericytes were identified by red fluorescence. Pericyte coverage of endothelial cells was determined by counting CD $31^{+}$cells in direct contact with desmin ${ }^{+}$cells in five randomly selected microscopic fields $(\times 200$ magnification $) .{ }^{24}$

\section{Quantification of Microvessel, CAF, and Collagen Areas}

To evaluate angiogenic activity of the tumors, microvessel $\left(\mathrm{CD} 31^{+}\right)$areas were quantified. Ten random fields at $\times 200$ magnification were captured for each tumor, and the vessels including a lumen were counted manually. The areas of CAFs and extracellular matrix (ECM) were also determined from the respective areas of $\alpha-\mathrm{SMA}^{+}$and type I collagen-positive staining from 10 optical fields $(\times 100$ magnification $)$ of different sections. The areas were calculated with the use of ImageJ software version 1.47v (NIH, Bethesda, MD).

\section{Determination of the Ki-67 Labeling Index and the Apoptotic Index}

The Ki-67 labeling index was determined by light microscopy at the site of the greatest number of $\mathrm{Ki}-67^{+}$cells. Cells were counted in 10 fields at $\times 200$ magnification, and the number of $\mathrm{Ki}-67^{+}$cells among approximately 300 tumor cells was counted and expressed as a percentage. Apoptotic cells were counted in 10 random $0.14-\mathrm{mm}^{2}$ fields at $\times 200$ magnification. The apoptotic index was taken as the ratio of positively stained tumor cells and bodies to all tumor cells and expressed as a percentage in each case.

\section{Statistical Analysis}

Between-group differences in murine body weight, tumor weight, and areas of $\alpha-\mathrm{SMA}^{+}$, type I collagen-positive, and $\mathrm{CD} 31^{+}$cells were analyzed by Wilcoxon/Kruskal-Wallis test. Differences in the incidence of lymph node metastasis were analyzed by Fisher's exact test. Differences in the percentages of Ki- $67^{+}$cells and TUNEL-positive cells were analyzed by unpaired Student's $t$-test or $\chi^{2}$ test as appropriate. $P<0.05$ was considered statistically significant. Data are expressed as means \pm SEM.

\section{Results}

\section{Expression of p-S6 in Human CRC Tissues and Orthotopic Xenografts in Nude Mice}

To determine whether the mTOR signaling pathway is activated in CRC tissues, we analyzed changes in expression of p-S6, a downstream effector of the mTOR signaling pathway, in 27 colon cancer tissues by immunohistochemistry (10 carcinomas in situ, 10 carcinomas showing submucosal invasion, and 7 carcinomas showing muscular invasion). Representative images are presented in Figure 1A. Normal colon mucosa was negative for p-S6 staining. Primary tumor cells exhibited diffuse cytoplasmic staining, regardless of disease stage. We also observed diffuse cytoplasmic staining of p-S6 in orthotopic xenografts derived from KM12C and KM12SM human CRC cells (Figure 1B). Phosphorylation of mTOR and S6 was confirmed by Western blot analysis in all four human CRC 
Table 1 Results of Treatment of KM12SM Tumors Growing in the Cecal Wall of Nude Mice

\begin{tabular}{|c|c|c|c|c|}
\hline Treatment group & $\begin{array}{l}\text { Body weight, g } \\
\text { (range) }\end{array}$ & $\begin{array}{l}\text { Tumor } \\
\text { incidence, } n / N\end{array}$ & $\begin{array}{l}\text { Tumor weight, } \\
\text { g (range) }\end{array}$ & $\begin{array}{l}\text { Lymph node } \\
\text { metastasis, } n / N\end{array}$ \\
\hline Control & $18.7(15.8-22.27)$ & $10 / 10$ & $0.45(0.21-1.52)$ & $7 / 10$ \\
\hline Nilotinib $(100 \mathrm{mg} / \mathrm{kg})$ & $19.3(15.7-22.04)$ & $9 / 9$ & $0.27(0.13-0.34)$ & $1 / 9$ \\
\hline Everolimus (1 mg/kg) & $19.2(17.79-20.13)$ & $10 / 10$ & $0.17(0.05-0.37)$ & $1 / 10$ \\
\hline Nilotinib $(100 \mathrm{mg} / \mathrm{kg})+$ everolimus $(1 \mathrm{mg} / \mathrm{kg})$ & $21.1(17.77-24.58)$ & $10 / 10$ & $0.05(0.02-0.08)$ & $0 / 10$ \\
\hline
\end{tabular}

cell lines (Figure 1C). These findings indicate that the activation of mTOR signaling is a widespread event in CRC.

\section{Effects of Everolimus and Nilotinib on the Growth of Colon Carcinoma Cells}

To assess the effects of everolimus and nilotinib on the growth of colon carcinoma cells in vitro, proliferation of KM12SM cells, both with and without the agents, was analyzed in real time. Growth curves revealed a dose-dependent decrease in the proliferation of KM12SM cells treated with everolimus (Figure 1D), but no inhibition of proliferation of KM12SM cells treated with an equivalent clinically effective dose of nilotinib (Figure 1E).

\section{Effects of Everolimus and Nilotinib on Phosphorylation of Targeted Proteins in KM12SM Cells}

To examine the effects of everolimus on mTOR signaling in vitro, Western blot analysis was performed on KM12SM cell lysate samples. S6 and mTOR phosphorylation was inhibited in a dose-dependent manner after 48 hours of everolimus treatment (Figure 2A). By contrast, phosphorylation of S6 and mTOR was not affected by treatment with nilotinib (Figure 2B). We next examined the effects of nilotinib on phosphorylation of PDGF-R $\beta$ in vitro. Because PDGF-R $\beta$ was not expressed in the colon cancer cell lines, MG63 cells (a human osteosarcoma cell line) were used as a positive control for PDGF-R expression. With nilotinib treatment, phosphorylation of PDGF-R $\beta$ in MG63 cells stimulated by PDGF-B was inhibited; with everolimus treatment, however, no effect on the PDGF pathways was observed in MG63 cells (Figure 2C). Furthermore, nilotinib did not influence the effect of everolimus on the mTOR pathway (Figure 2C).

\section{Effects of Treatment on KM12SM Tumors Growing in the Cecal Wall of Nude Mice}

We next determined whether tumor growth at the primary site is inhibited by nilotinib, everolimus, or the two agents administered in combination (Table 1). Tumor incidence was $100 \%$ in all four treatment groups. Tumor weights were measured at necropsy (Figure 3A). Tumor weight was significantly reduced in the group treated with everolimus $(P<0.01)$. Tumor weight tended to decrease in the group treated with nilotinib, but the difference was not statistically significant. Tumor weight of the mice treated with both everolimus and nilotinib was much lower than that of the mice treated with vehicle alone, and also lower than that of mice treated with everolimus alone $(P<0.01)$. Lymph node metastasis was significantly inhibited in the three agent-treated groups. There was no significant difference between these three treatment groups, although lymph node metastasis was not found in the combination treatment group. There was no significant change in body weight in the combination group, but cachexia-associated weight loss occurred in the other two agent-treated groups and in the control group (Figure 3B).

Effects of Nilotinib and Everolimus on PDGF and mTOR Signaling Pathways in Orthotopic Tumors

To assess whether nilotinib or everolimus inhibits phosphorylation of targeted proteins, tumor specimens were analyzed immunohistochemically for expression of p-PDGF-R and p-S6. Phosphorylation of PDGF-R in the stroma was significantly inhibited in orthotopic tumors of mice treated with nilotinib alone or with nilotinib and everolimus in combination (Figure 4B). Phosphorylation of S6 ribosomal protein in the
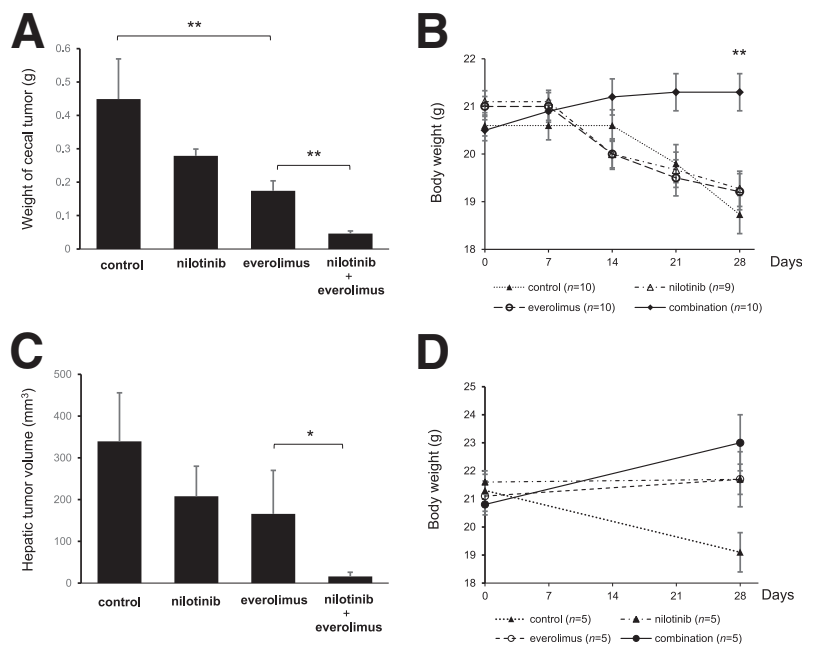

Figure 3 Effects of nilotinib and/or everolimus on KM12SM orthotopically implanted (cecal) tumors (A and $\mathbf{B}$ ) and on liver metastasis (C and $\mathbf{D})$. Treatment with combination therapy significantly reduced the weight of primary tumors (A) and the volume of metastatic tumors (C). Increased body weight of mice was observed only in the combination treatment group; control mice developed cachexia with tumor progression (B and $\mathbf{D})$. Data are expressed as means \pm SEM. ${ }^{*} P<0.05 ;{ }^{*} P<0.01$. 


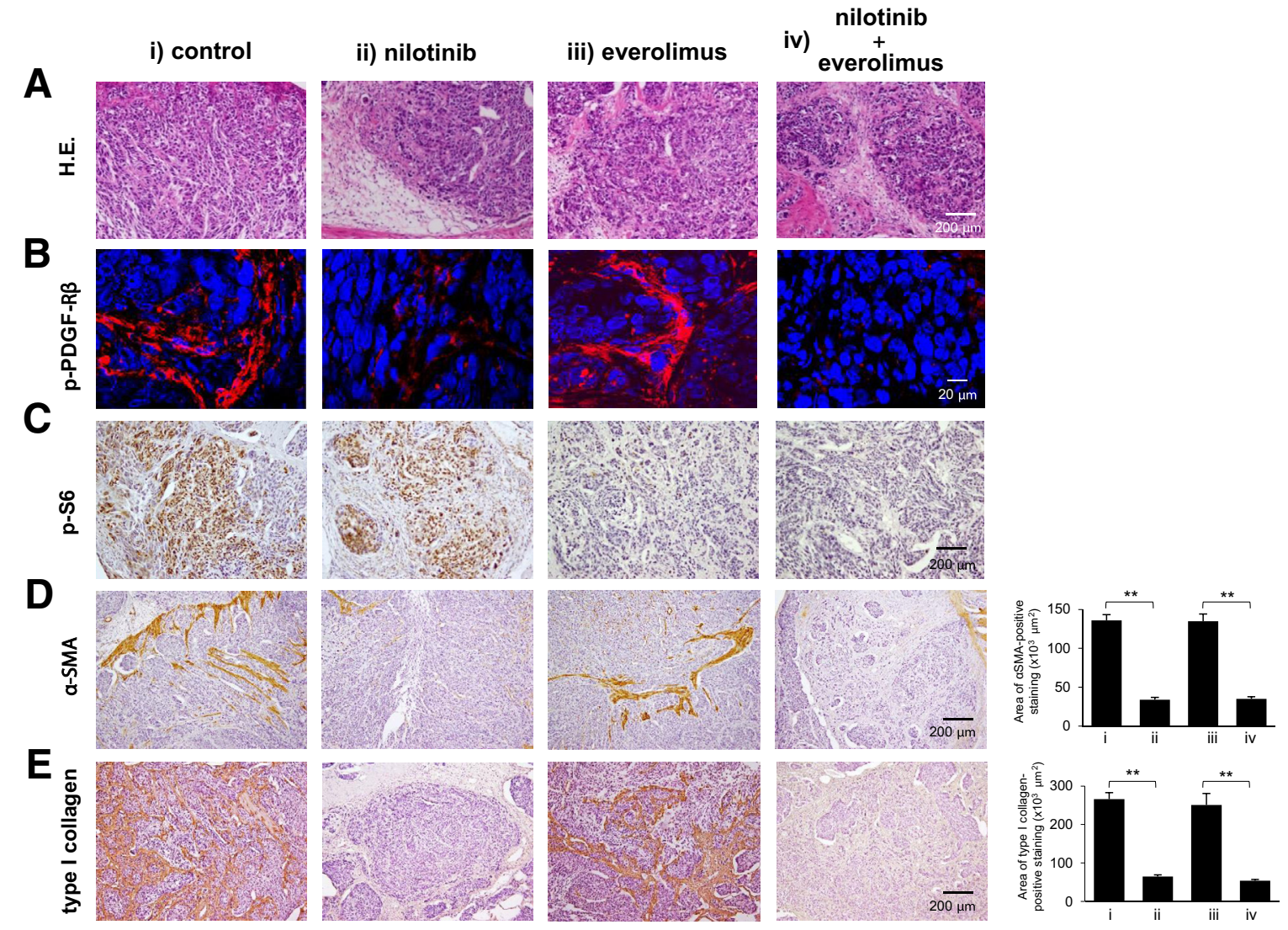

Figure 4 Histological and immunofluorescence staining for p-PDGF-R $\beta$ (red fluorescence), p-S6, $\alpha$-SMA, and type I collagen. Mice with cecal KM12SM tumors were treated with i) vehicle (control), ii) nilotinib, iii) everolimus, or iv) nilotinib and everolimus. A: Hematoxylin and eosin (H.E.) stain. B: Phosphorylation of PDGF-R $\beta$ in stroma was inhibited by treatment with nilotinib and nilotinib and everolimus in combination. C: Phosphorylation of S6 in tumor nests was inhibited by treatment with everolimus and by combination therapy. D and E: The stromal reaction, evaluated by immunostaining for $\alpha$-SMA and type I collagen, was significantly reduced in mice treated with nilotinib and with combination therapy, compared with the control and everolimus groups. Data are expressed as means \pm SEM. ${ }^{* *} P<0.01$. Scale bars: $200 \mu \mathrm{m}(\mathbf{A}, \mathbf{C}-\mathbf{E}) ; 20 \mu \mathrm{m}$ (B).

tumor cells was inhibited in orthotopic tumors of mice treated with everolimus alone or with everolimus and nilotinib in combination (Figure 4C). Inhibition of mTOR signaling by treatment with everolimus was confirmed by Western blot analysis (Figure 2D).

\section{Histopathological Analysis of KM12SM Cecal Tumors}

KM12SM cells orthotopically implanted into the cecum of nude mice grew invasively, as aggressive tumors with a stromal reaction. Under nilotinib treatment, the stromal reaction was reduced, and tumors grew expansively. With everolimus treatment, an active stromal reaction was observed, and tumors grew invasively. Under treatment with nilotinib and everolimus in combination, the inhibitory effect of nilotinib on the stromal reaction was maintained (Figure 4A). The stromal reaction in tumors was evaluated as the area of $\alpha$-SMA or type I collagen positivity. The stromal reaction was significantly reduced in mice treated with nilotinib, compared with that in the control group, but in mice treated with everolimus the stromal reaction was unchanged (Figure 4, D and E).
The Ki-67 labeling index was used as a marker of cell proliferation (Figure 5A). The labeling index decreased under treatment with everolimus alone and under treatment with everolimus and nilotinib in combination; however, there was no decrease in the labeling index in cecal tumors under treatment with nilotinib alone. To determine whether the drug treatment is associated with cellular apoptosis, colonic tumors were examined by TUNEL assay. In tumors from control mice, the median number of TUNEL-positive tumor cells was only $2.1 \pm 0.64$. The TUNEL-positive cells increased in number in tumors from agent-treated mice, with no statistically significant difference between the three treatments (Figure 5B). The CD31 index was used as a marker for microvessel density. We found reduced microvessel density with everolimus treatment but no change in microvessel density with nilotinib treatment. Everolimus and nilotinib in combination yielded the greatest decrease in microvessel density (Figure 5C). The difference in microvessel density between everolimus treatment and combination treatment was significant $(P=0.028)$. We also examined expression of the angiogenic factor VEGF-A. Immunoreactivity of VEGF-A in tumor cells was markedly 


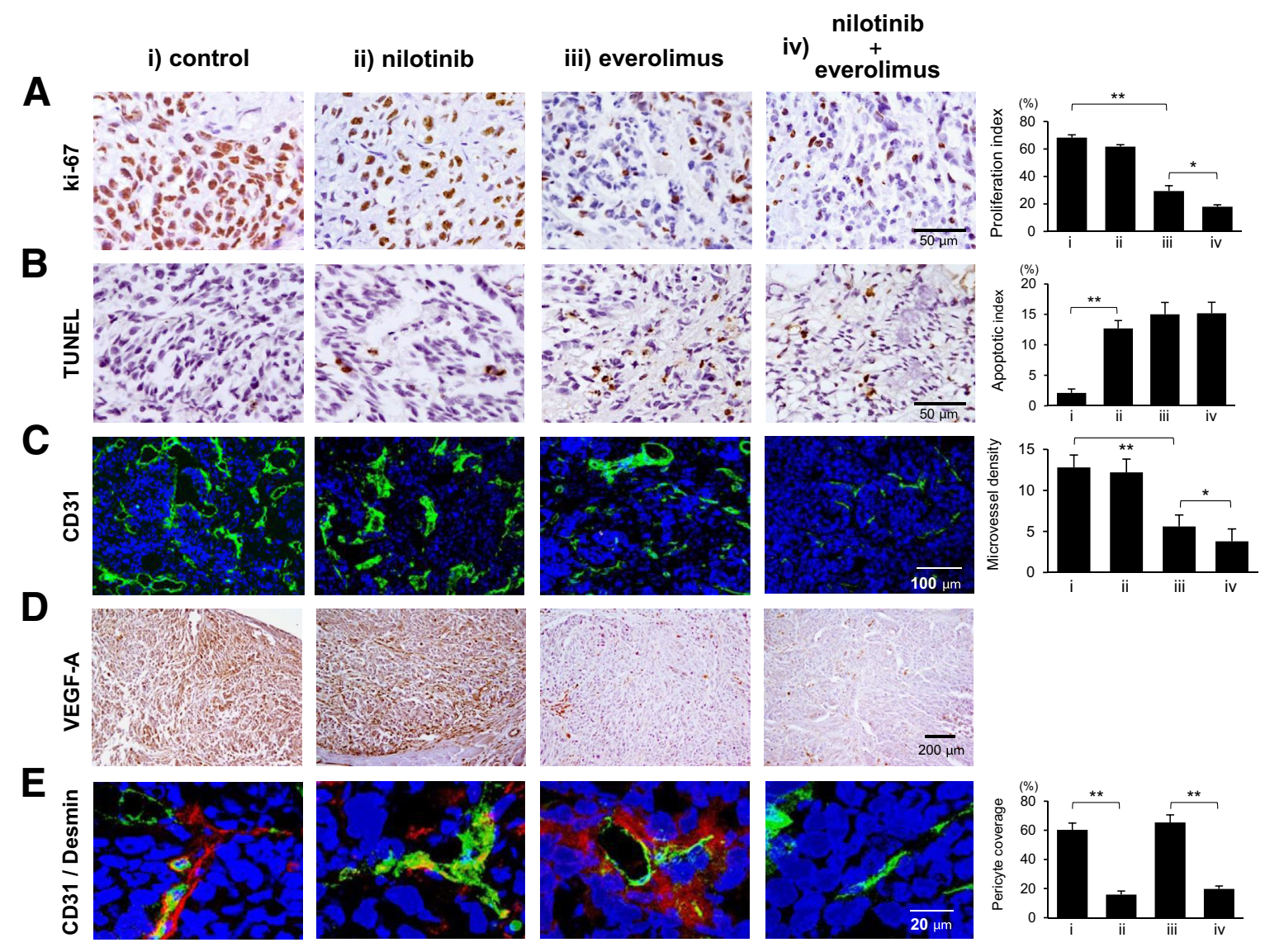

Figure 5 Effects of nilotinib and/or everolimus on KM12SM orthotopic tumors. Analysis of cell proliferation (Ki-67) (A), apoptosis (TUNEL) (B), angiogenesis (CD31 and VEGF-A) (C and D), and pericyte coverage of microvessels (CD31, desmin) (E). Vascular endothelial cells were identified by green fluorescence and pericytes by red fluorescence. Data are expressed as means \pm SEM. ${ }^{*} P<0.05 ;{ }^{*} P<0.01$. Scale bars: $200 \mu \mathrm{m}(\mathbf{D}) ; 100 \mu \mathrm{m}(\mathbf{C}) ; 50 \mu \mathrm{m}(\mathbf{A}$ and $\mathbf{B})$; $20 \mu \mathrm{m}(\mathbf{E})$.

suppressed under treatment with everolimus (Figure 5D). The effect of nilotinib treatment on pericyte coverage of tumorassociated endothelial cells was evaluated by double immunofluorescence staining with anti-CD31 and anti-desmin antibodies. The number of pericytes covering endothelial cells decreased in the nilotinib treatment group (Figure 5E).

\section{Effects of Treatment on KM12SM Tumors Growing in the Liver of Nude Mice}

We next examined the effects of nilotinib, everolimus, and the two agents in combination on growth of KM12SM cells in the experimental liver metastasis model (Table 2). Both the number and volume of liver metastatic tumors decreased remarkably under combination therapy $(P<0.05)$ (Figure 3C). The number of metastatic foci and total volume of the liver tumors tended to decrease under treatment with each agent alone, but the difference was not significant. In two of the five mice in the combination group, no metastatic liver tumor was observed macroscopically, and no loss of body weight was observed in the combination group (Table 2). Indeed, mice in the combination group tended to gain weight (Figure 3D).

\section{Histopathological Analysis of KM12SM Metastatic Liver Tumors}

A marked stromal reaction was observed in the metastatic KM12SM tumors in the mouse livers. In the control group,

Table 2 Results of Treatment of KM12SM Tumors Growing in the Liver of Nude Mice

\begin{tabular}{|c|c|c|c|c|}
\hline Treatment group & $\begin{array}{l}\text { Body weight, } \\
\text { g (range) }\end{array}$ & $\begin{array}{l}\text { Tumor } \\
\text { incidence, } n / N\end{array}$ & $\begin{array}{l}\text { Hepatic tumor } \\
\text { volume, } \mathrm{mm}^{3} \text { (range) }\end{array}$ & $\begin{array}{l}\text { Number of colonies } \\
\text { (range) }\end{array}$ \\
\hline Control & $19.6(17.0-21.05)$ & $5 / 5$ & $339.5(9.5-703)$ & $13(4-14)$ \\
\hline Everolimus (1 mg/kg) & $21.7(20.0-23.0)$ & $5 / 5$ & $165.7(6.5-575.5)$ & $6.2(1-14)$ \\
\hline Nilotinib $(100 \mathrm{mg} / \mathrm{kg})+$ everolimus $(1 \mathrm{mg} / \mathrm{kg})$ & $23.0(19.95-26.05)$ & $3 / 5$ & $15.8(4-53.5)$ & $1.6(1-4)$ \\
\hline
\end{tabular}




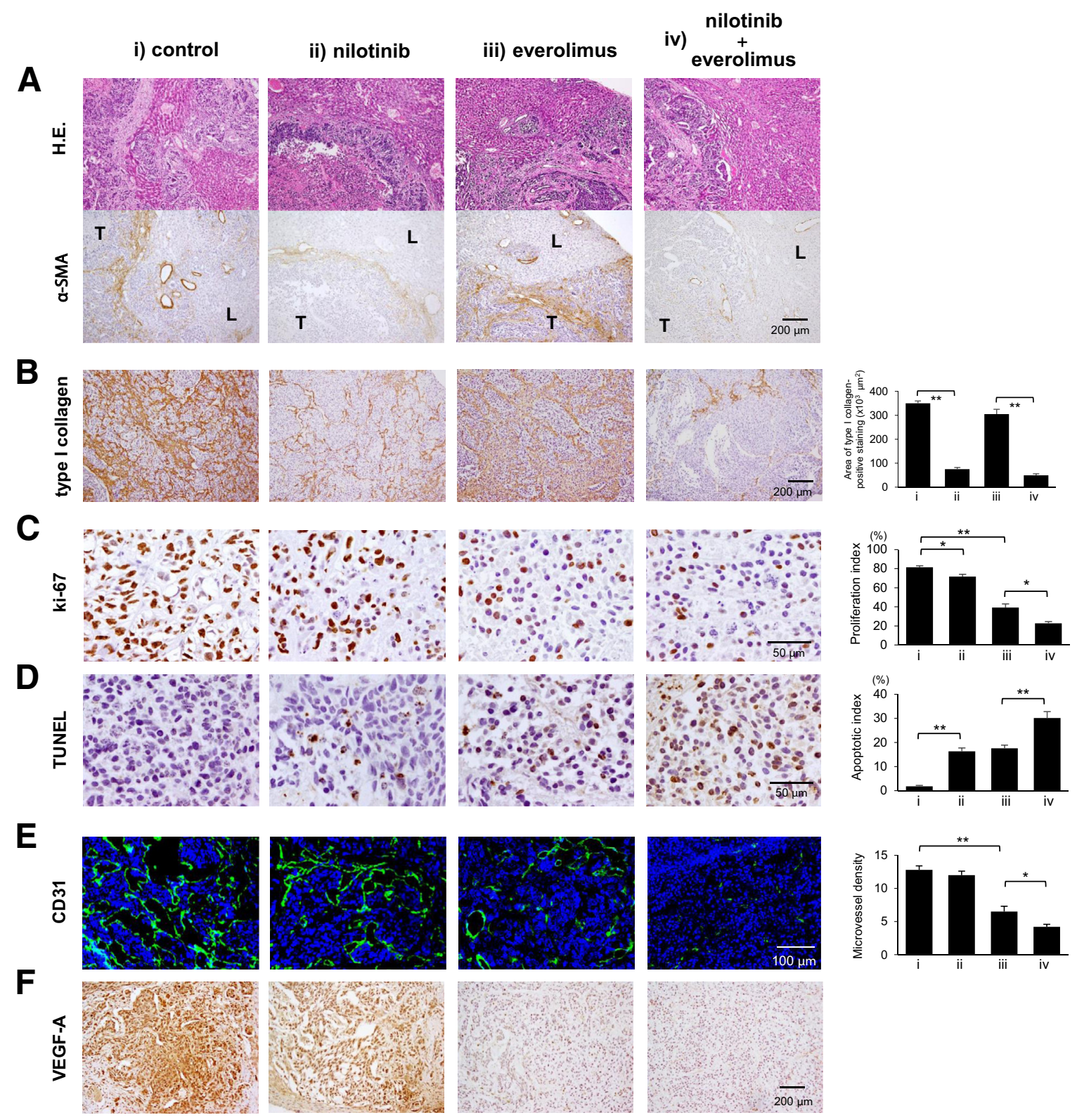

Figure 6 Effects of nilotinib and/or everolimus on KM12SM metastatic liver tumors. A: Histological and immunohistochemical staining for $\alpha$-SMA at the periphery of metastatic tumors; within groups, image pairs depict the same lesion. B: Immunohistochemical staining for type I collagen at the center of metastatic tumors. C-F: Analysis of cell proliferation (Ki-67), apoptosis (TUNEL), and angiogenesis (CD31 and VEGF-A). Vascular endothelial cells were identified by green fluorescence. ${ }^{*} P<0.05 ;{ }^{*} P<0.01$. Scale bars: $200 \mu \mathrm{m}(\mathbf{A}, \mathbf{B}$, and $\mathbf{F}) ; 100 \mu \mathrm{m}(\mathbf{E}) ; 50 \mu \mathrm{m}(\mathbf{C}$ and $\mathbf{D})$. L, liver tissue; T, metastatic tumor.

tumor cells had infiltrated into the activated stroma, especially at the border zone between the metastatic tumor nest and normal tissue. The same infiltration was observed in mice treated with everolimus (Figure 6A). A reduction in stromal reaction was observed in mice treated with nilotinib or with combination therapy. No change in stromal reaction was observed in mice treated with everolimus alone (Figure 6B). The Ki-67 labeling index decreased and the number of TUNEL-positive cells increased under treatment with everolimus alone, and these effects were remarkably greater in the combination group (Figure 6, C and D). Microvessel density decreased with everolimus, but did not change with nilotinib. The change in microvessel density was most pronounced with everolimus and nilotinib in combination (Figure 6E), and the difference in microvessel density between everolimus treatment and combination treatment was statistically significant $(P=0.023)$. Immunoreactivity of VEGF-A in metastatic liver tumor cells was markedly suppressed under treatment with everolimus (Figure 6F).

\section{Discussion}

Here, we have shown that nilotinib (a PDGF-R tyrosine kinase inhibitor) reduces stromal reaction and that everolimus (an mTOR inhibitor) decreases proliferation and 
increases apoptosis of tumor cells. In combination, these molecular-target drugs significantly inhibited tumor growth, stromal reaction, and angiogenesis not only at the site of orthotopic implantation (cecum), but also at the site of metastasis (liver).

Expression of PDGF-R in stroma has been reported to be associated with a poor prognosis in several types of cancer, including breast, prostate, and pancreatic cancers. ${ }^{25,26} \mathrm{We}$ have previously reported that PDGF-R is phosphorylated in the stroma of human colon cancer tissues and that increased expression levels are associated with tumor invasion and metastasis. $^{7}$ PDGF-B is expressed by cancer cells, whereas PDGF-R $\beta$ is expressed mainly by stromal cells, including CAFs and pericytes. ${ }^{11,23,27}$ Thus, PDGF-R expressing stroma may be involved in crosstalk with malignant cells that promotes growth and invasion, and thus metastasis. Consistent with our previous studies of treatment with imatinib (another PDGF-R tyrosine kinase inhibitor), ${ }^{6}$ treatment with nilotinib decreased the stromal reaction and number of pericytes of cecal tumors and liver metastases. However, the inhibitory effect on tumor growth was minimal with administration of nilotinib alone, whether in vitro or in vivo.

Here, we have shown that activation of mTOR signaling occurs even in early-stage human colon cancer tissues, and confirm that everolimus actually inhibits phosphorylation of mTOR in vitro and in vivo. mTOR inhibition has been shown to decrease neovascularization via inhibition of VEGF expression by tumor cells. ${ }^{28-30}$ In accord with earlier reports, everolimus inhibited tumor growth and VEGF expression, leading to significant inhibition of angiogenesis in tumor stroma. ${ }^{28-30}$

Pericyte recruitment is part of the development of normal functional capillaries. ${ }^{31}$ Previous studies have shown that the tumor-associated microvasculature in human colon carcinoma tissues contains multiple pericytes, which may protect endothelial cells against antiangiogenic therapy. ${ }^{32,33}$ Targeting VEGF receptors that act primarily on immature blood vessels has attracted attention as a novel approach to antiangiogenic therapy. ${ }^{34,35}$ Because nilotinib inhibits pericyte coverage of tumor vasculature and everolimus inhibits VEGF expression by tumor cells, combination therapy with these drugs may produce synergistic antivascular effects.

To date, most preclinical studies of chemotherapy have been performed in subcutaneous cell transplant models. However, the orthotopic organ microenvironment has been shown to influence the stromal reaction, cell differentiation, and drug resistance. ${ }^{6}$ We therefore used an orthotopic model for the present study. In clinical situations, the primary tumor and regional lymph nodes of most patients with advanced-stage CRC would be surgically resected, regardless of the presence of liver metastasis, to avoid colonic obstruction and bleeding from tumor tissues. Although complete resection can eliminate tumor cells from the primary site, microscopic metastasis might have already occurred. Inhibition of tumor cell growth and invasion in the liver is therefore the most important focus of adjuvant chemotherapy. In the present study, metastatic liver tumors had a remarkable stromal reaction, like that of the primary cecal tumors. Tumor nests were surrounded by active stroma into which tumor cells infiltrated. No fibroblasts reside in the liver, and sources of CAFs in metastatic liver tumor have not been fully clarified. We have previously reported that bone marrow-derived mesenchymal stem cells migrate to liver metastatic sites and differentiate to CAFs. Imatinib inhibits migration and differentiation of mesenchymal stem cells, and so inhibits stromal reaction. ${ }^{27,36}$ Increased interstitial fluid pressure in solid tumors, which is mediated by an increase in the stromal compartment, acts as a barrier between tumor cells and normal tissues against effective distribution of antitumor drugs. ${ }^{37}$ The PDGF-R signaling pathway plays a critical role in the control of interstitial fluid pressure. ${ }^{37,38}$ Inhibition of PDGF-R signaling can decrease this pressure and enhance the effects of simultaneously used chemotherapeutic agents. ${ }^{10}$ Because treatment with nilotinib reduces the stromal reaction and interstitial fluid pressure, everolimus can be easily delivered to tumor tissues. Thus, the combination of nilotinib and everolimus had synergistic effects on tumor growth and angiogenesis by inhibition of mTOR, VEGF, and PDGF signaling.

In summary, nilotinib-everolimus combination therapy targeting both tumor cells and stromal components resulted in a remarkable inhibition of tumor growth at both the primary site and metastatic site. This is the first report to show a promising effect on CRC of combination therapy with both a PDGF-R tyrosine kinase inhibitor and an mTOR inhibitor.

\section{Acknowledgments}

This work was performed with the kind cooperation of the Analysis Center of Life Science and Institute of Laboratory Animal Science (Hiroshima University, Hiroshima, Japan). We thank Novartis Pharma K.K. (Tokyo, Japan) for everolimus and nilotinib and Dr. Isaiah J. Fidler (MD Anderson Cancer Center, University of Texas, Houston, TX) for human colon cancer cell lines KM12SM and KM12C.

\section{References}

1. Jemal A, Bray F, Center MM, Ferlay J, Ward E, Forman D: Global cancer statistics [Erratum appeared in CA Cancer J Clin 2011, 61:134]. CA Cancer J Clin 2011, 61:69-90

2. Wolpin BM, Mayer RJ: Systemic treatment of colorectal cancer. Gastroenterology 2008, 134:1296-1310

3. Whiteside TL: The tumor microenvironment and its role in promoting tumor growth. Oncogene 2008, 27:5904-5912

4. Mantovani A, Allavena P, Sica A, Balkwill F: Cancer-related inflammation. Nature 2008, 454:436-444

5. Mueller MM, Fusenig NE: Friends or foes-bipolar effects of the tumour stroma in cancer. Nat Rev Cancer 2004, 4:839-849 
6. Kitadai Y, Sasaki T, Kuwai T, Nakamura T, Bucana CD, Fidler IJ: Targeting the expression of platelet-derived growth factor receptor by reactive stroma inhibits growth and metastasis of human colon carcinoma. Am J Pathol 2006, 169:2054-2065

7. Kitadai Y, Sasaki T, Kuwai T, Nakamura T, Bucana CD, Hamilton SR, Fidler IJ: Expression of activated platelet-derived growth factor receptor in stromal cells of human colon carcinomas is associated with metastatic potential. Int J Cancer 2006, 119:2567-2574

8. Ostman A: PDGF receptors-mediators of autocrine tumor growth and regulators of tumor vasculature and stroma. Cytokine Growth Factor Rev 2004, 15:275-286

9. Bergers G, Song S, Meyer-Morse N, Bergsland E, Hanahan D: Benefits of targeting both pericytes and endothelial cells in the tumor vasculature with kinase inhibitors. J Clin Invest 2003, 111:1287-1295

10. Pietras K: Increasing tumor uptake of anticancer drugs with imatinib. Semin Oncol 2004, 31:18-23

11. Onoyama M, Kitadai Y, Tanaka Y, Yuge R, Shinagawa K, Tanaka S, Yasui W, Chayama K: Combining molecular targeted drugs to inhibit both cancer cells and activated stromal cells in gastric cancer. Neoplasia 2013, 15:1391-1399

12. Roy HK, Olusola BF, Clemens DL, Karolski WJ, Ratashak A, Lynch HT, Smyrk TC: AKT proto-oncogene overexpression is an early event during sporadic colon carcinogenesis. Carcinogenesis 2002, 23:201-205

13. Philp AJ, Campbell IG, Leet C, Vincan E, Rockman SP, Whitehead RH, Thomas RJ, Phillips WA: The phosphatidylinositol 3'kinase p85alpha gene is an oncogene in human ovarian and colon tumors. Cancer Res 2001, 61:7426-7429

14. Guertin DA, Sabatini DM: Defining the role of mTOR in cancer. Cancer Cell 2007, 12:9-22

15. Lane HA, Wood JM, McSheehy PM, Allegrini PR, Boulay A, Brueggen J, Littlewood-Evans A, Maira SM, Martiny-Baron G, Schnell CR, Sini P, O'Reilly T: mTOR inhibitor RAD001 (everolimus) has antiangiogenic/vascular properties distinct from a VEGFR tyrosine kinase inhibitor. Clin Cancer Res 2009, 15:1612-1622

16. Mabuchi S, Altomare DA, Cheung M, Zhang L, Poulikakos PI, Hensley HH, Schilder RJ, Ozols RF, Testa JR: RAD001 inhibits human ovarian cancer cell proliferation, enhances cisplatin-induced apoptosis, and prolongs survival in an ovarian cancer model. Clin Cancer Res 2007, 13:4261-4270

17. Mabuchi S, Altomare DA, Connolly DC, Klein-Szanto A, Litwin S, Hoelzle MK, Hensley HH, Hamilton TC, Testa JR: RAD001 (everolimus) delays tumor onset and progression in a transgenic mouse model of ovarian cancer. Cancer Res 2007, 67:2408-2413

18. Manegold PC, Paringer C, Kulka U, Krimmel K, Eichhorn ME, Wilkowski R, Jauch KW, Guba M, Bruns CJ: Antiangiogenic therapy with mammalian target of rapamycin inhibitor RAD001 (everolimus) increases radiosensitivity in solid cancer. Clin Cancer Res 2008, 14: 892-900

19. Shinohara ET, Cao C, Niermann K, Mu Y, Zeng F, Hallahan DE, Lu B: Enhanced radiation damage of tumor vasculature by mTOR inhibitors. Oncogene 2005, 24:5414-5422

20. Morikawa K, Walker SM, Nakajima M, Pathak S, Jessup JM, Fidler IJ: Influence of organ environment on the growth, selection, and metastasis of human colon carcinoma cells in nude mice. Cancer Res 1988, 48:6863-6871

21. Kim SJ, Uehara H, Karashima T, Shepherd DL, Killion JJ, Fidler IJ: Blockade of epidermal growth factor receptor signaling in tumor cells and tumor-associated endothelial cells for therapy of androgenindependent human prostate cancer growing in the bone of nude mice. Clin Cancer Res 2003, 9:1200-1210

22. Deininger MW: Nilotinib. Clin Cancer Res 2008, 14:4027-4031

23. Sumida T, Kitadai Y, Shinagawa K, Tanaka M, Kodama M, Ohnishi M, Ohara E, Tanaka S, Yasui W, Chayama K: Anti-stromal therapy with imatinib inhibits growth and metastasis of gastric carcinoma in an orthotopic nude mouse model. Int J Cancer 2011, 128 2050-2062

24. Yokoi K, Sasaki T, Bucana CD, Fan D, Baker CH, Kitadai Y, Kuwai T, Abbruzzese JL, Fidler IJ: Simultaneous inhibition of EGFR, VEGFR, and platelet-derived growth factor receptor signaling combined with gemcitabine produces therapy of human pancreatic carcinoma and prolongs survival in an orthotopic nude mouse model. Cancer Res 2005, 65:10371-10380

25. Hägglöf C, Hammarsten P, Josefsson A, Stattin P, Paulsson J, Bergh A, Ostman A: Stromal PDGFRbeta expression in prostate tumors and non-malignant prostate tissue predicts prostate cancer survival. PLoS One 2010, 5:e10747

26. Paulsson J, Sjöblom T, Micke P, Pontén F, Landberg G, Heldin CH, Bergh J, Brennan DJ, Jirström K, Ostman A: Prognostic significance of stromal platelet-derived growth factor beta-receptor expression in human breast cancer. Am J Pathol 2009, 175:334-341

27. Shinagawa $K$, Kitadai $Y$, Tanaka $M$, Sumida $T$, Onoyama $M$, Ohnishi M, Ohara E, Higashi Y, Tanaka S, Yasui W, Chayama K: Stroma-directed imatinib therapy impairs the tumor-promoting effect of bone marrow-derived mesenchymal stem cells in an orthotopic transplantation model of colon cancer. Int $\mathrm{J}$ Cancer 2013, 132: $813-823$

28. Guba M, von Breitenbuch P, Steinbauer M, Koehl G, Flegel S, Hornung M, Bruns CJ, Zuelke C, Farkas S, Anthuber M, Jauch KW, Geissler EK: Rapamycin inhibits primary and metastatic tumor growth by antiangiogenesis: involvement of vascular endothelial growth factor. Nat Med 2002, 8:128-135

29. Guba M, Yezhelyev M, Eichhorn ME, Schmid G, Ischenko I, Papyan A, Graeb C, Seeliger H, Geissler EK, Jauch KW, Bruns CJ: Rapamycin induces tumor-specific thrombosis via tissue factor in the presence of VEGF. Blood 2005, 105:4463-4469

30. O'Reilly T, McSheehy PM: Biomarker development for the clinical activity of the mTOR inhibitor everolimus (RAD001): processes, limitations, and further proposals. Transl Oncol 2010, 3:65-79

31. Gerhardt H, Betsholtz C: Endothelial-pericyte interactions in angiogenesis. Cell Tissue Res 2003, 314:15-23

32. Pietras K, Hanahan D: A multitargeted, metronomic, and maximumtolerated dose "chemo-switch" regimen is antiangiogenic, producing objective responses and survival benefit in a mouse model of cancer. J Clin Oncol 2005, 23:939-952

33. Erber R, Thurnher A, Katsen AD, Groth G, Kerger H, Hammes HP, Menger MD, Ullrich A, Vajkoczy P: Combined inhibition of VEGF and PDGF signaling enforces tumor vessel regression by interfering with pericyte-mediated endothelial cell survival mechanisms. FASEB J 2004, 18:338-340

34. Hurwitz H, Fehrenbacher L, Novotny W, Cartwright T, Hainsworth J, Heim W, Berlin J, Baron A, Griffing S, Holmgren E, Ferrara N, Fyfe G, Rogers B, Ross R, Kabbinavar F: Bevacizumab plus irinotecan, fluorouracil, and leucovorin for metastatic colorectal cancer. $\mathrm{N}$ Engl J Med 2004, 350:2335-2342

35. Bergers G, Benjamin LE: Tumorigenesis and the angiogenic switch. Nat Rev Cancer 2003, 3:401-410

36. Shinagawa K, Kitadai Y, Tanaka M, Sumida T, Kodama M, Higashi Y, Tanaka S, Yasui W, Chayama K: Mesenchymal stem cells enhance growth and metastasis of colon cancer. Int J Cancer 2010, $127: 2323-2333$

37. Pietras K, Östman A, Sjöquist M, Buchdunger E, Reed RK, Heldin $\mathrm{CH}$, Rubin K: Inhibition of platelet-derived growth factor receptors reduces interstitial hypertension and increases transcapillary transport in tumors. Cancer Res 2001, 61:2929-2934

38. Hwang RF, Yokoi K, Bucana CD, Tsan R, Killion JJ, Evans DB, Fidler IJ: Inhibition of platelet-derived growth factor receptor phosphorylation by STI571 (Gleevec) reduces growth and metastasis of human pancreatic carcinoma in an orthotopic nude mouse model. Clin Cancer Res 2003, 9:6534-6544 